\title{
Treatment of a cerebral pial arteriovenous fistula in a patient with sickle cell disease-related moyamoya syndrome: case report
}

\author{
Anna Lo Presti, MD, ${ }^{1}$ Alexander G. Weil, MD, ${ }^{1}$ Aria Fallah, MD, MSc, ${ }^{1}$ Eric C. Peterson, MD, ${ }^{2}$ \\ Toba N. Niazi, MD, ${ }^{1}$ and Sanjiv Bhatia, MD1
}

1Division of Pediatric Neurosurgery, Department of Neurosurgery, Miami Children's Hospital, University of Miami/Miller School of Medicine; 'Division of Neuroendovascular Surgery, Department of Neurosurgery, University of Miami/Miller School of Medicine, Miami, Florida

\begin{abstract}
Sickle cell disease (SCD) is an autosomal recessive hematological disorder, characterized by sickling of the red blood cells in response to a hypoxic stress and vaso-occlusive crises. It is associated with moyamoya-like changes on cerebral angiographic imaging in $43 \%$ of patients. Cerebral aneurysms, arteriovenous malformations, and dural arteriovenous fistulas (AVFs) have been described in association with SCD and moyamoya disease. However, the description of a pial AVF (pAVF) in a patient with SCD and/or moyamoya formation has not yet been reported. The authors present the case of a 15-year-old boy with SCD-associated moyamoya disease harboring a pAVF who developed a de novo venous aneurysm 8 months after undergoing indirect superficial temporal artery-middle cerebral artery (MCA) bypass that was complicated by bilateral ischemia of the MCA territory. The pAVF was successfully treated with transarterial embolization using Onyx. The authors describe the possible pathophysiological mechanisms and management strategies for this rare occurrence.
\end{abstract}

http://thejns.org/doi/abs/10.3171/2014.12.PEDS14486

KEY WORDS sickle cell anemia; moyamoya syndrome; pial arteriovenous fistula; encephaloduroarteriosynangiosis; Onyx; vascular disorders

$\mathrm{S}$ ICKLE cell disease (SCD) is a hereditary, autosomal recessive disorder, characterized by vaso-occlusive crises that may affect multiple organs, and is associated with a wide variety of cerebral vascular complications. ${ }^{11,20}$ Up to $43 \%$ of patients with SCD who have suffered a stroke or transient ischemic attack before 18 years of age will demonstrate moyamoya-like changes on angiographic imaging. ${ }^{6,11}$ Cerebral aneurysms, arteriovenous malformations (AVMs) and dural arteriovenous fistulas $(\mathrm{dAVFs})^{10,16}$ have been reported to occur spontaneously and following revascularization surgery in patients with SCD and moyamoya disease. ${ }^{3-5,7,10,13,16,21,28-30,33}$ However, to our knowledge, a pial arteriovenous fistula (pAVF) has never been reported to occur in a patient with SCD and moyamoya disease. We report on a 15 -year-old boy with SCD and moyamoya syndrome who harbored a pAVF and developed a de novo venous varix/aneurysm that subsequently ruptured 8 months after bilateral indirect STA (superficial temporal artery)-MCA (middle cerebral artery) bypass. The objective is to demonstrate 1) that pAVFs may develop spontaneously in patients with moyamoya disease, 2) that they may become symptomatic, and 3) that treatment of these lesions is feasible with transarterial embolization using Onyx. We discuss the possible pathophysiological mechanisms of and management strategy for this rare occurrence.

ABBREVIATIONS AED = antiepileptic drug; AVF = arteriovenous fistula; $A V M=$ arteriovenous malformation; $d A V F=$ dural AVF; HHT = hemorrhagic hereditary telangiectasia; ICA = internal carotid artery; MCA = middle cerebral artery; PAVF = pial AVF; SCD = sickle cell disease; STA = superficial temporal artery.

SUBMITTED September 15, 2014. ACCEPTED December 31, 2014.

INCLUDE WHEN CITING Published online May 22, 2015; DOI: 10.3171/2014.12.PEDS14486.

DISCLOSURE The authors report no conflict of interest concerning the materials or methods used in this study or the findings specified in this paper. 


\section{Case Report}

History and Examination

A 14-year-old boy with a history of SCD and a left MCA territory stroke at 4 years of age (resulting in right hemiparesis, speech changes, and seizures) presented with new-onset left hemiparesis. At the time of presentation, he was being treated with serial blood transfusions and antiepileptic drugs (AEDs). MRI of the brain showed a porencephalic region in the left frontal region and late post-ischemic changes in the watershed area in the right hemisphere. An angiogram showed complete obliteration of the right supraclinoid internal carotid artery (ICA) and stenosis of the left supraclinoid ICA with very poor recruitment from the external circulation and active recruitment from the posterior cerebral vessels, leading to the diagnosis of Suzuki Stage 3 moyamoya syndrome. Although it was not appreciated initially, a direct pAVF arising from a right MCA lenticulostriate artery and draining in a periventricular vein draining toward the internal cerebral vein was visible as shown on MRI (Fig. 1A-C) and angiography (Fig. 1D). These findings, although not initially evident, were identified when the imaging studies were re-evaluated after the patient presented with the symptomatic pAVF varix hemorrhage.

\section{Surgery and Postoperative Course}

The patient underwent bilateral encephaloduroarteriosynangiosis after preoperative exchange transfusion and hydration. Postoperatively, he developed seizures. An MRI obtained on the 1st postoperative day revealed acute ischemia involving bilateral frontocentral regions and thalami (Fig. 1E) which was treated with hydration and exchange transfusions. He made a good recovery following rehabilitation but had persistent mild left hemiparesis (strength 4+/5). He had no recurrence of stroke or seizures on maintenance aspirin therapy and AEDs.

Eight months following bilateral revascularization surgery, the patient presented with a decreased level of con-
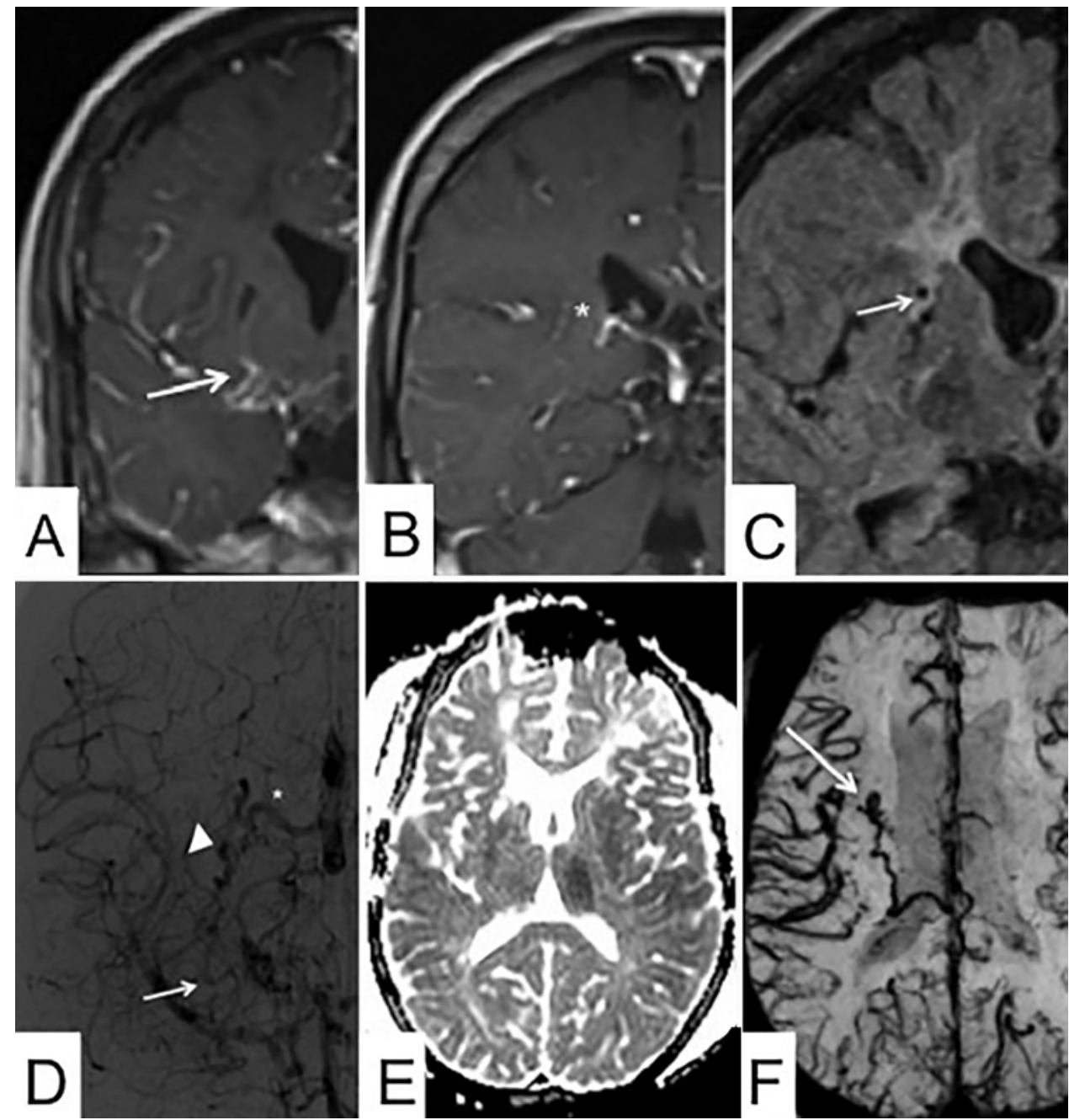

FIG. 1. A and B: Gadolinium-enhanced T1-weighted coronal MR images revealing a right basal ganglia pAVF fed by a medial lenticulostriate artery (white arrow) and draining into a periventricular vein (asterisk). C: Coronal FLAIR MR image showing subcortical ischemic changes at the site of the pAVF. D: Anteroposterior angiography showing the pAVF. E and F: Diffusion-weighted (E) and $T 2^{*}$-weighted $(F)$ MR images obtained after bilateral pial synangiosis showing bilateral ischemia affecting the left hemisphere more than the right and the persistent pAVF with a possible small aneurysm ( $F$, white arrow). 
sciousness and left hemibody and facial paresis. A CT scan of the brain demonstrated a large round hyperdense lesion in the right basal ganglia associated with vasogenic edema (Fig. 2A). The patient's hemoglobin level was $8 \mathrm{~g} / \mathrm{dl}$. He was treated with blood transfusion and hydration in the intensive care unit. MR angiography and CT angiography were performed, demonstrating a large, partially thrombosed aneurysm arising from the previously missed pAVF (Fig. 2B-D). Catheter angiography revealed a persistently occluded right ICA. Injection of the left internal carotid artery and left vertebral artery demonstrated cross flow to the right MCA via the posterior communicating artery. An enlarged lateral lenticulostriate artery was visualized, which drained into a large venous varix finally draining into the deep venous system (Fig. 2E and F). Given the presence of a prior right craniotomy with dependence on the external carotid artery (middle meningeal artery and STA) for vascularization of the right hemisphere, the decision was made to avoid a craniotomy and attempt endovascular embolization of the lesion.

\section{Endovascular Procedure}

After the diagnostic angiogram was performed, a 5-F sheath was placed into the right common femoral artery and the child was heparinized to an activated clotting time of 250-300 seconds. A 5-F Envoy guiding catheter (DePuy Synthes) was navigated into the left vertebral artery, and a 3D rotational angiogram was performed. A Marathon microcatheter (Covidien) was navigated over a Mirage microwire (Covidien) into the posterior circulation, across the right posterior communicating artery, and into the right MCA. The microcatheter was then carefully navigated into the enlarged lenticulostriate artery feeding the fistula, and a superselective angiogram was performed, which demonstrated the fistulous anatomy (Fig. 3). The microcatheter was flushed with dimethyl sulfox- ide, and under negative roadmapping technique, Onyx-18 (Covidien) was slowly injected into the fistula. The material propagated through the fistula into the draining vein and varix. A small amount of reflux was seen into the lenticulostriate artery, but not in the $\mathrm{M}_{1}$ trunk. The catheter was removed, and a follow-up angiogram was performed, which demonstrated complete obliteration of the fistula.

\section{Postoperative Course}

Postoperatively, the patient demonstrated gradual improvement in his left-sided paresis. He was discharged to a neurological rehabilitation center. At last follow-up, 6 months after embolization, his left hemibody strength had significantly improved.

\section{Discussion}

To our knowledge, this is the first reported case of a pAVF in a patient with SCD and moyamoya syndrome. The case is unique in that a venous varix/aneurysm developed de novo and became symptomatic following an indirect STA-MCA bypass procedure that was complicated by cerebral ischemia in the area of the pAVF.

Pial AVFs are rare cerebrovascular lesions characterized by an AVF without an intervening nidus. ${ }^{37}$ They differ from AVMs, which are always associated with a nidus. ${ }^{17}$ They can also be confused with micro-AVMs, but micro-AVMs also have a discernable, albeit small $(<1$ $\mathrm{cm}$ ) nidus. ${ }^{2}$ They are also distinct from dAVFs, which lie within dural leaflets and may have cortical drainage..$^{22,27}$

The occurrence of a pAVF in this patient is likely related to the proangiogenic environment found in moyamoya arteriopathy, in SCD, and after cerebral ischemia. ${ }^{14-16,26,29,33,35}$

In general, although pAVFs can be acquired following trauma or sinus thrombosis, ${ }^{24}$ the majority of cases appear to be congenital and may be associated with hem-

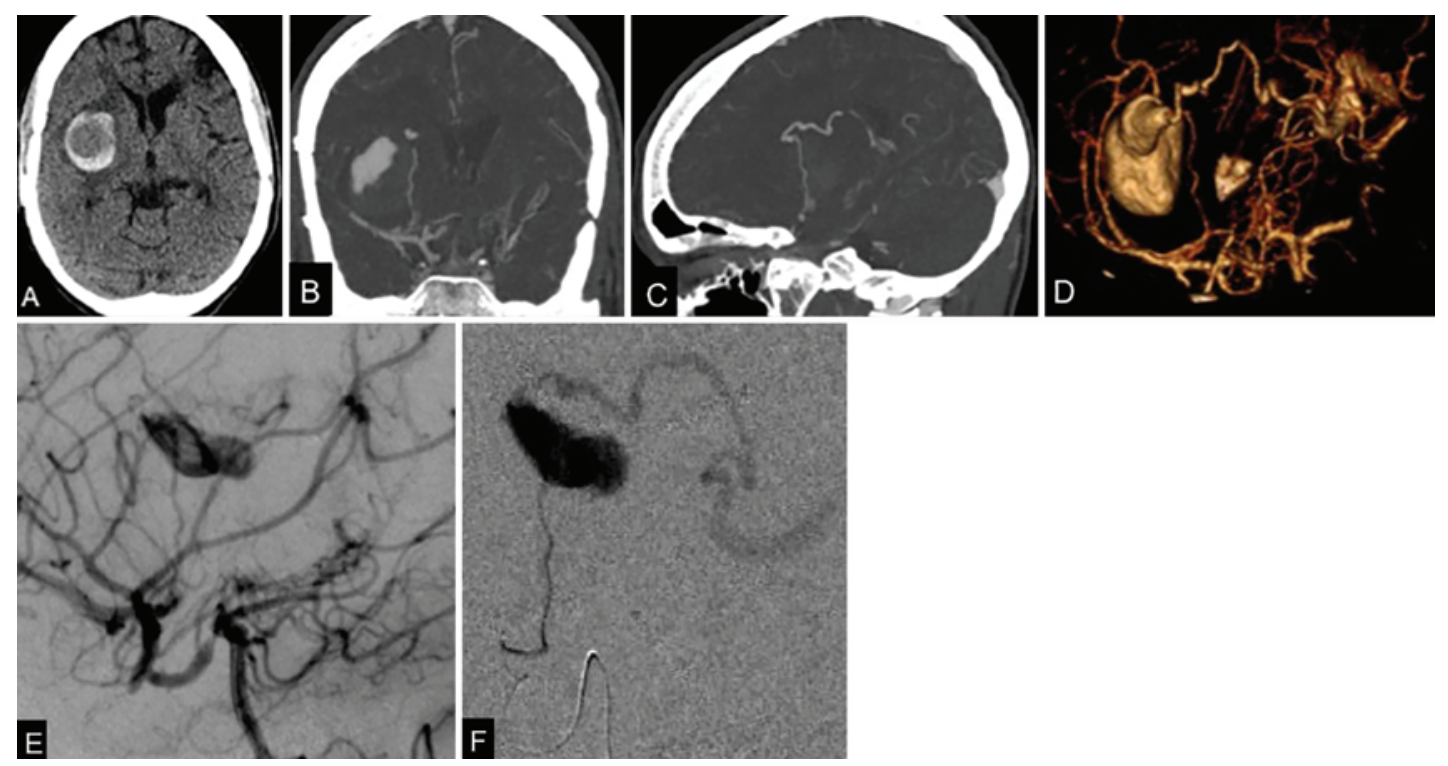

FIG. 2. A: CT scan obtained at presentation showing a spontaneously partially thrombosed aneurysm within the right basal ganglia associated with significant cerebral edema. B-D: CT angiograms confirming the persistent right pAVF and partially thrombosed aneurysm. E and F: Angiograms obtained with right vertebral artery (E) and supraselective right MCA (F) injection showing the pAVF fed by the right medial lenticulostriate and draining in the deep vein, associated with an aneurysmal dilation. Figure is available in color online only. 


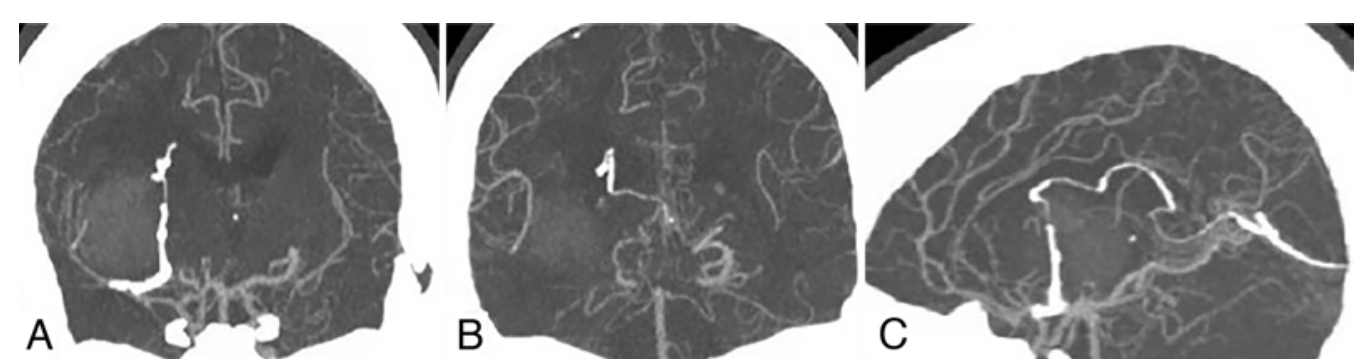

FIG. 3. CT angiogram obtained after embolization showing Onyx material filling the length of the pAVF with some reflux into the straight sinus and complete thrombosis of the aneurysm.

orrhagic hereditary telangiectasia (HHT) and capillary malformation (CM)-AVM/RASAl gene mutation syndrome. ${ }^{12,23}$ Although rare, there are multiple reports describing an association between moyamoya disease and both AVMs ${ }^{1,8,9,12,21,25,26,34}$ and dAVFs. ${ }^{10,16}$ While most report the coexistence of an $\mathrm{AVM}^{1,8,9,15,21,25,26,34}$ or dAVF ${ }^{16}$ and moyamoya arteriopathy, suggesting that the AVM or dAVF, may lead to moyamoya syndrome, ${ }^{25}$ three reports have actually demonstrated de novo appearance of an $\mathrm{AVM}^{29,33}$ or $\mathrm{dAVF}^{10}$ in patients with moyamoya arteriopathy, one of whom also had $\mathrm{SCD}^{29}$ and two of whom had a previous infarct in the exact location of the eventual arteriovenous malformation. ${ }^{10,33}$ Elevated levels of angiogenic basic fibroblast growth factor (bFGF) have been reported in patients with moyamoya disease,$^{35}$ and we hypothesize that the combination of such an increased level of bFGF and local angiogenic stimulation due to cerebral infarction may have been involved in the development of this patient's pAVF. ${ }^{33,34}$ Although there is the distinct possibility that the pAVF and moyamoya arteriopathy in our patient may coexist independently, the occurrence of the pAVF in an area of focal subcortical ischemia (Fig. 1D) and its being fed by a lenticulostriate vessel, known to collaterize ischemic areas in moyamoya syndrome, suggest that they are related.

In our patient, the venous varix developed within 8 months of indirect vascularization. It is unclear if the pAVF should have been addressed at the outset, prior to revascularization. A review of the angiogram done at that time showed that a superselective angiographic intervention would have been very difficult. It is well known that the fistulous communication between a feeding artery and a single draining vein can produce venous dilation, varices, and even aneurysms through turbulent high flow. 13,18,36 Because pediatric cases of PAVF have a high tendency for varix formation, ${ }^{31}$ and patients often become symptomatic with neurological deficit, seizure, or symptoms due to AVF rupture, prophylactic treatment of our patient's pAVF might have been warranted. However, both pial ${ }^{32}$ and HHT-related ${ }^{19}$ AVFs have been reported to regress spontaneously. The rarity of these lesions renders any conclusion regarding natural history speculative.

Treatment strategies for pial AVFs include microsurgery and endovascular techniques.,10,28 The majority of pediatric patients with pAVFs described in the literature have been treated with endovascular embolization ${ }^{22,37}$ Furthermore, in the setting of moyamoya arteriopathy and SCD, where cerebral vascularization is dependent on collaterals arising from external carotid scalp and dural arteries, a craniotomy would carry a high risk of cerebral ischemia. The vast majority of prior AVMs in patients with moyamoya have been treated via an endovascular approach for this reason..$^{22}$ Very few cases of pAVFs embolized with Onyx have been reported in literature. ${ }^{37}$ Some authors have advised against using nonadherent embolic agents, such as Onyx, to treat fistulous lesions because Onyx utilization requires more fluoroscopic time in children and the potentially large doses required for embolization of these fistulas increase the risk for dimethyl sulfoxide-related pulmonary toxicity associated with Onyx, which may exacerbate the preexisting cardiovascular and pulmonary comorbidities common in this group of children. ${ }^{22}$ Newman et al., published the first successful balloon-assisted Onyx embolization of a giant, high-flow pAVF, noticing the increased ability to control the delivery of Onyx, as compared with NBCA glue, allowing more precise targeting and delivery of embolic material to the fistulous point. ${ }^{27}$ In our case, we were able to specifically target the fistula with no reflux into the MCA, which was only a few millimeters away. In addition, despite the fact that the route to the target was complicated by the extensive arteriopathy, modern endovascular techniques allow superselective catheterization-in this case, from the posterior circulation via the posterior communicating artery.

\section{Conclusions}

Like AVMs and dAVFs, a pAVF may form in rare cases of moyamoya syndrome due to the inherent proangiogenic environment and the angiogenic effects of local ischemia. The management of these AVFs is complicated by the presence of intracranial arteriopathy and limited endovascular access. A craniotomy should be avoided in order to avoid disrupting collateral blood flow from the external carotid artery. Endovascular transarterial embolization with Onyx is a feasible alternative.

\section{References}

1. Akiyama K, Minakawa T, Tsuji Y, Isayama K: Arteriovenous malformation associated with moyamoya disease: case report. Surg Neurol 41:468-471, 1994

2. Alén JF, Lagares A, Paredes I, Campollo J, Navia P, Ramos A, et al: Cerebral microarteriovenous malformations: a series of 28 cases. J Neurosurg 119:594-602, 2013

3. Batjer HH, Adamson TE, Bowman GW: Sickle cell disease and aneurysmal subarachnoid hemorrhage. Surg Neurol 36:145-149, 1991

4. Chalouhi N, Tjoumakaris S, Gonzalez LF, Dumont AS, Shah Q, Gordon D, et al: Onyx embolization of a ruptured lenticulostriate artery aneurysm in a patient with moyamoya disease. World Neurosurg 80:436.e7-436.e10, 2013 
5. Cheatham ML, Brackett CE: Problems in management of subarachnoid hemorrhage in sickle cell anemia. J Neurosurg 23:488-493, 1965

6. Dobson SR, Holden KR, Nietert PJ, Cure JK, Laver JH, Disco D, et al: Moyamoya syndrome in childhood sickle cell disease: a predictive factor for recurrent cerebrovascular events. Blood 99:3144-3150, 2002

7. Fujimura M, Kimura N, Ezura M, Niizuma K, Uenohara H, Tominaga T: Development of a de novo arteriovenous malformation after bilateral revascularization surgery in a child with moyamoya disease. J Neurosurg Pediatr 13:647-649, 2014

8. Fuse T, Takagi T, Fukushima T, Hashimoto N, Yamada K: Arteriovenous malformation associated with moyamoya disease. Childs Nerv Syst 12:404-408, 1996

9. Halatsch ME, Rustenbeck HH, Jansen J: Progression of arteriovenous malformation in moyamoya syndrome. Acta Neurochir (Wien) 139:82-85, 1997

10. Hanaoka M, Matsubara S, Satoh K, Nagahiro S: Dural arteriovenous fistulae after cerebral infarction: report of two cases. Neurosurgery 68:E575-E580, 2011

11. Hankinson TC, Bohman LE, Heyer G, Licursi M, Ghatan S, Feldstein NA, et al: Surgical treatment of moyamoya syndrome in patients with sickle cell anemia: outcome following encephaloduroarteriosynangiosis. J Neurosurg Pediatr 1:211-216, 2008

12. Herbert SP, Huisken J, Kim TN, Feldman ME, Houseman BT, Wang RA, et al: Arterial-venous segregation by selective cell sprouting: an alternative mode of blood vessel formation. Science 326:294-298, 2009

13. Hoh BL, Putman CM, Budzik RF, Ogilvy CS: Surgical and endovascular flow disconnection of intracranial pial singlechannel arteriovenous fistulae. Neurosurgery 49:1351-1364, 2001

14. Hoshimaru M, Takahashi JA, Kikuchi H, Nagata I, Hatanaka M: Possible roles of basic fibroblast growth factor in the pathogenesis of moyamoya disease: an immunohistochemical study. J Neurosurg 75:267-270, 1991

15. Kayama T, Suzuki S, Sakurai Y, Nagayama T, Ogawa A, Yoshimoto T: A case of moyamoya disease accompanied by an arteriovenous malformation. Neurosurgery 18:465-468, 1986

16. Killory BD, Gonzalez LF, Wait SD, Ponce FA, Albuquerque FC, Spetzler RF: Simultaneous unilateral moyamoya disease and ipsilateral dural arteriovenous fistula: case report. Neurosurgery 62:E1375-E1376, 2008

17. Lasjaunias $P$, Manelfe C, Chiu M: Angiographic architecture of intracranial vascular malformations and fistulas-pretherapeutic aspects. Neurosurg Rev 9:253-263, 1986

18. Lawton MT, Jacobowitz R, Spetzler RF: Redefined role of angiogenesis in the pathogenesis of dural arteriovenous malformations. J Neurosurg 87:267-274, 1997

19. Leung KM, Agid R, terBrugge K: Spontaneous regression of a cerebral arteriovenous malformation in a child with hereditary hemorrhagic telangiectasia. Case report. J Neurosurg 105 (5 Suppl):428-431, 2006

20. Liaquat I, Murphy M, Bassi S, Bullock PR: Paediatric and adult vascular intracranial complications of sickle-cell disease. Acta Neurochir (Wien) 152:1175-1179, 2010

21. Lichtor T, Mullan S: Arteriovenous malformation in moyamoya syndrome. Report of three cases. J Neurosurg 67:603-608, 1987

22. Madsen PJ, Lang SS, Pisapia JM, Storm PB, Hurst RW, Heuer GG: An institutional series and literature review of pial arteriovenous fistulas in the pediatric population. Clinical article. J Neurosurg Pediatr 12:344-350, 2013

23. Mao GH, Wang QH: Transvenous embolization with Onyx for a cerebral arteriovenous fistula originating from the distal lenticulostriate artery. Br J Neurosurg 27:532-534, 2013
24. Matsubara S, Satoh K, Satomi J, Shigekiyo T, Kinouchi T, Miyake H, et al: Acquired pial and dural arteriovenous fistulae following superior sagittal sinus thrombosis in patients with protein S deficiency: a report of two cases. Neurol Med Chir (Tokyo) 54:245-252, 2014

25. Mawad ME, Hilal SK, Michelsen WJ, Stein B, Ganti SR: Occlusive vascular disease associated with cerebral arteriovenous malformations. Radiology 153:401-408, 1984

26. Montanera W, Marotta TR, terBrugge KG, Lasjaunias P, Willinsky R, Wallace MC: Cerebral arteriovenous malformations associated with moyamoya phenomenon. AJNR Am J Neuroradiol 11:1153-1156, 1990

27. Newman CB, Hu YC, McDougall CG, Albuquerque FC: Balloon-assisted Onyx embolization of cerebral single-channel pial arteriovenous fistulas. J Neurosurg Pediatr 7:637-642, 2011

28. Okada T, Kida Y, Kinomoto T, Sakurai T, Kobayashi T: Arteriovenous malformation associated with moyamoya disease-case report. Neurol Med Chir (Tokyo) 30:945-948, 1990

29. O'Shaughnessy BA, DiPatri AJ Jr, Parkinson RJ, Batjer HH: Development of a de novo cerebral arteriovenous malformation in a child with sickle cell disease and moyamoya arteriopathy. Case report. J Neurosurg 102 (2 Suppl):238-243, 2005

30. Overby MC, Rothman AS: Multiple intracranial aneurysms in sickle cell anemia. Report of two cases. J Neurosurg 62:430-434, 1985

31. Panigrahi S, Mishra SS, Das S, Parida DK: Intracerebral pial arteriovenous fistula with large venous varix: A rare case report. Surg Neurol Int 4:22, 2013

32. Santosh C, Teasdale E, Molyneux A: Spontaneous closure of an intracranial middle cerebral arteriovenous fistula. Neuroradiology 33:65-66, 1991

33. Schmit BP, Burrows PE, Kuban K, Goumnerova L, Scott RM: Acquired cerebral arteriovenous malformation in a child with moyamoya disease. Case report. J Neurosurg 84:677-680, 1996

34. Scott RM: Arteriovenous malformation and moyamoya disease. Childs Nerv Syst 13:357, 1997 (Letter)

35. Takahashi A, Sawamura Y, Houkin K, Kamiyama H, Abe $\mathrm{H}$ : The cerebrospinal fluid in patients with moyamoya disease (spontaneous occlusion of the circle of Willis) contains high level of basic fibroblast growth factor. Neurosci Lett 160:214-216, 1993

36. Viñuela F, Drake CG, Fox AJ, Pelz DM: Giant intracranial varices secondary to high-flow arteriovenous fistulae. J Neurosurg 66:198-203, 1987

37. Walcott BP, Smith ER, Scott RM, Orbach DB: Pial arteriovenous fistulae in pediatric patients: associated syndromes and treatment outcome. J Neurointerv Surg 5:10-14, 2013

\section{Author Contributions}

Conception and design: Bhatia, Lo Presti, Weil. Acquisition of data: Lo Presti, Weil. Analysis and interpretation of data: Bhatia, Lo Presti, Weil. Drafting the article: Lo Presti, Weil. Critically revising the article: Fallah, Peterson, Niazi. Reviewed submitted version of manuscript: Bhatia, Fallah, Peterson, Niazi.

\section{Correspondence}

Sanjiv Bhatia, Division of Pediatric Neurosurgery, Miami Children's Hospital, Department of Neurosurgery, University of Miami/Miller School of Medicine, 3100 Ambulatory Care Bldg., SW 62nd Ave., Miami, FL 33155. email: sbhatia@med.miami. edu. 